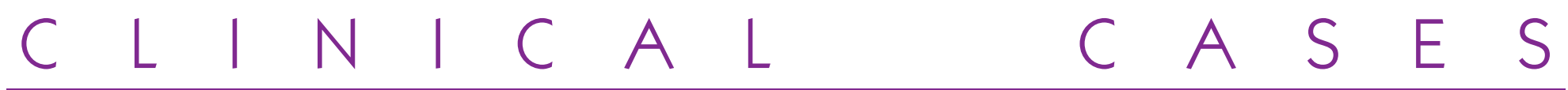

\title{
Treatment of a case of hyperdivergence showing contributions of myo-functional therapy
}

Jean-Marie LANDAU

M-A. B., born 3/08/1995

came to see me for the first time in October 2005.

DIAGNOSIS (fig. 1 to 5 )

Cl. II Ballard, skeletal hyperdivergence.

Cl. II, div 1 Angle, arch length discrepancy.

This patient has serious functional problems having been a thumb sucker until a late age. As a result she retained a primitive swallowing pattern, was a mouth breather, and kept her tongue in a low position.
Because of this patient's numerous functional problems that could have a negative influence on the development of her entire facial complex, we decided to begin treatment with a stage of myofunctional therapy aimed at eliminating noxious habits little by little and at the same time providing us with an indication of how good her cooperation was going to be.

\section{TREATMENT}

\section{Phase I}

Patient wore a "T4K ${ }^{@}$ Position trainer for 6 months.

At that time we re-evaluated her status (fig 6 to 7).

\section{Results :}

- patient regained lip competence and the ridges around the nose diminished, indicating that nasal breathing had improved ; 


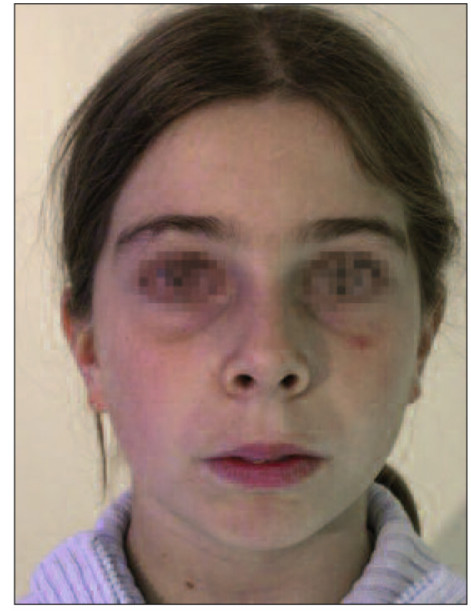

a

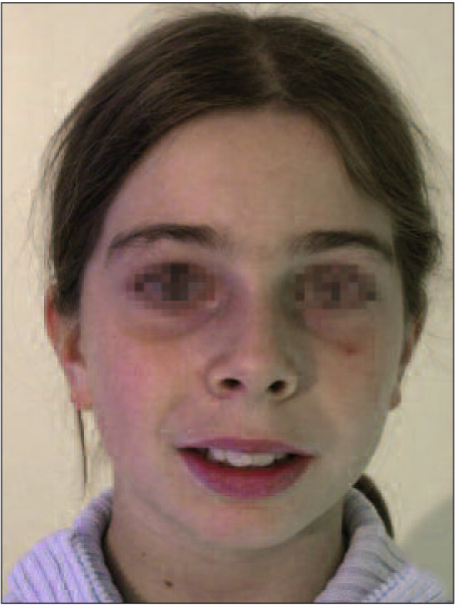

$b$

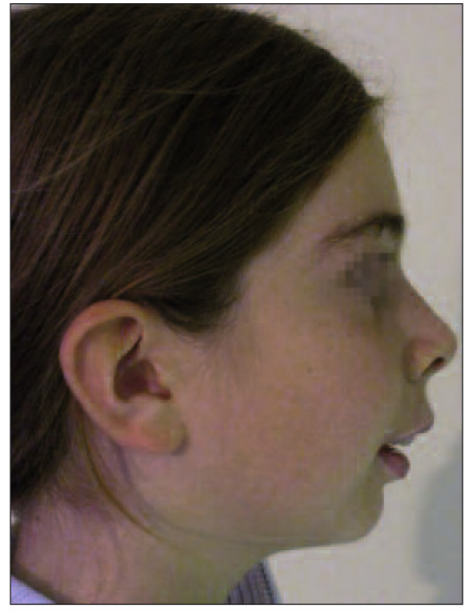

C

Figures 1 a to $c$

Pretreatment facial photos.

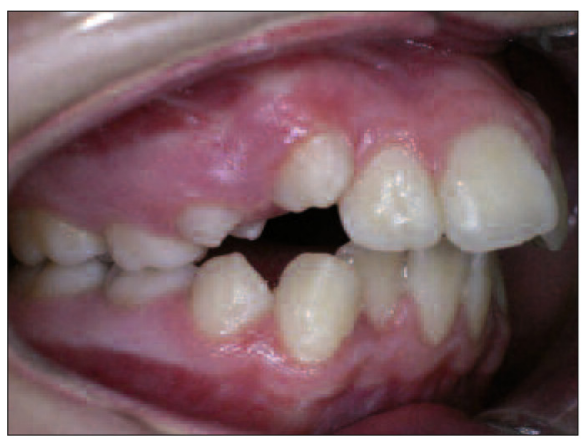

a

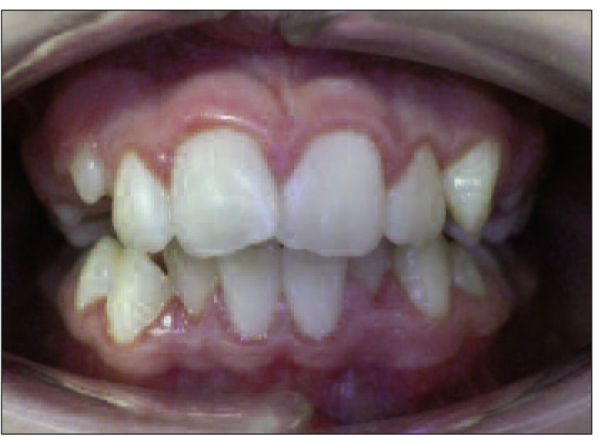

$b$

Figures 2 a to $c$

Pretreatment intraoral views.

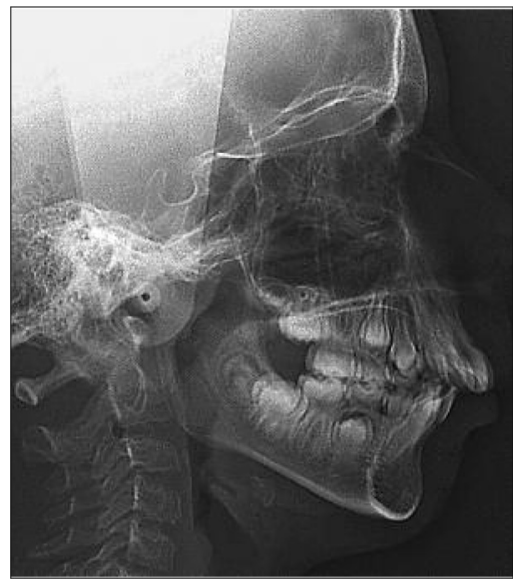

Figure 3

Pretreatment profile cephalometric $X$-ray.

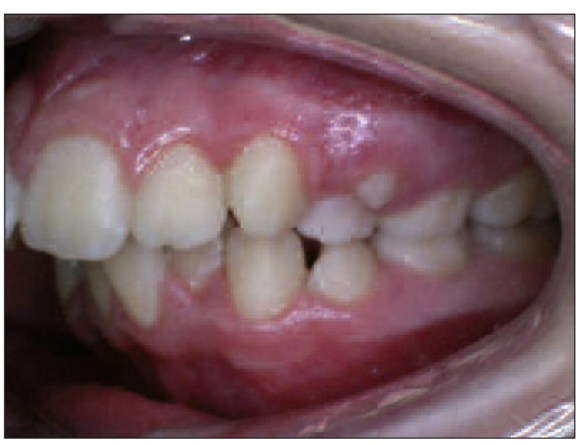

C

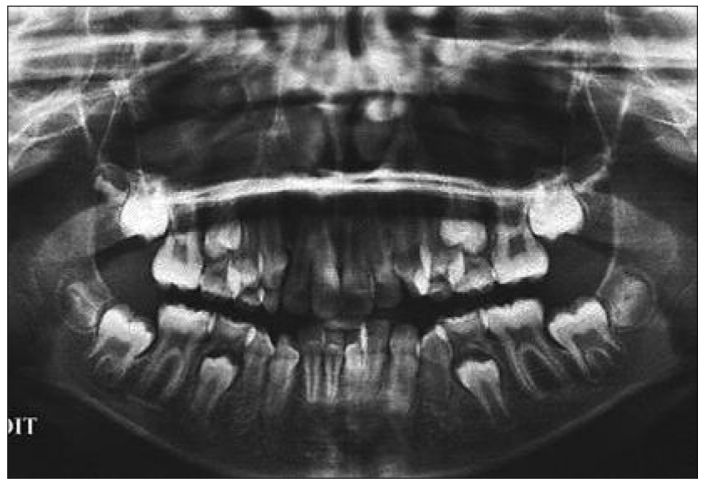

Figure 4

Pretreatment panoramic $X$-ray film.

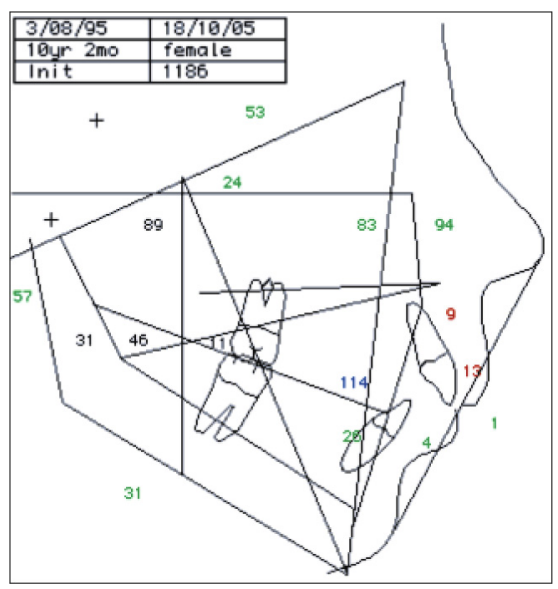

Figure 5

Pretreatment cephalometric tracing. 

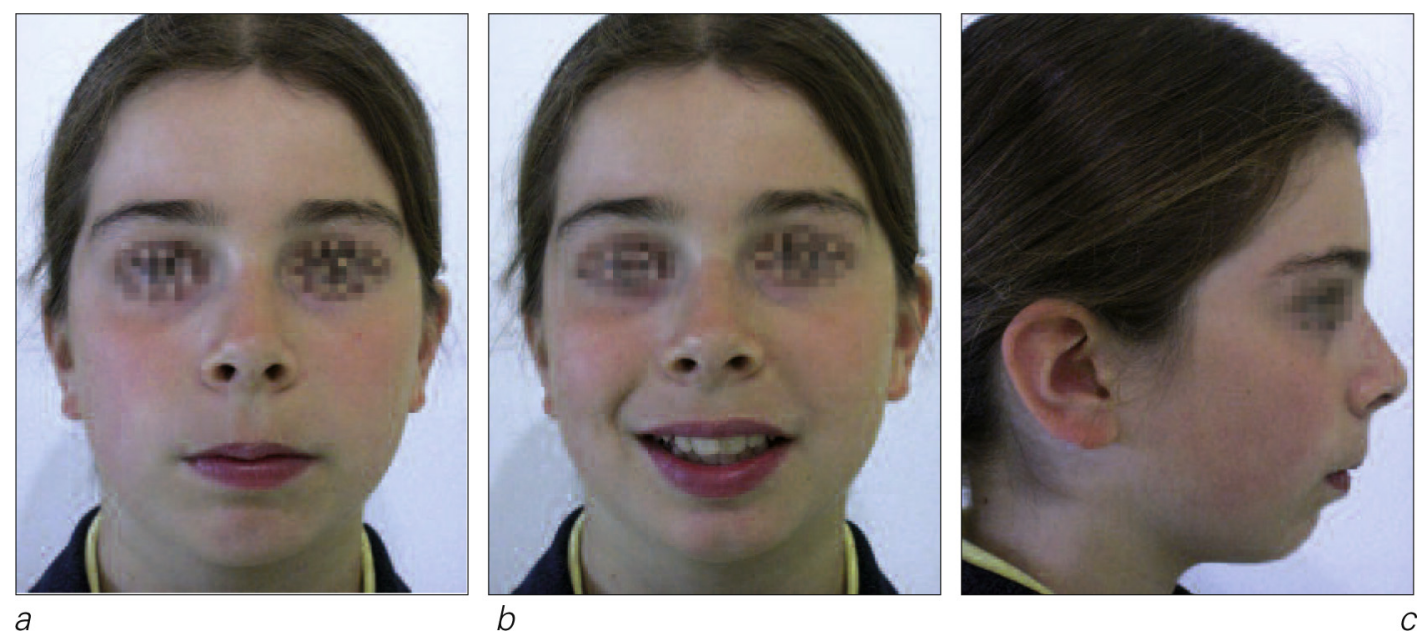

Figures 6 a to $c$

Facial photos at the end of the first stage of treatment.

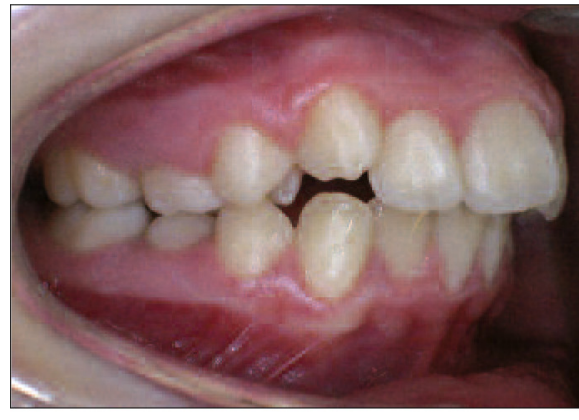

a

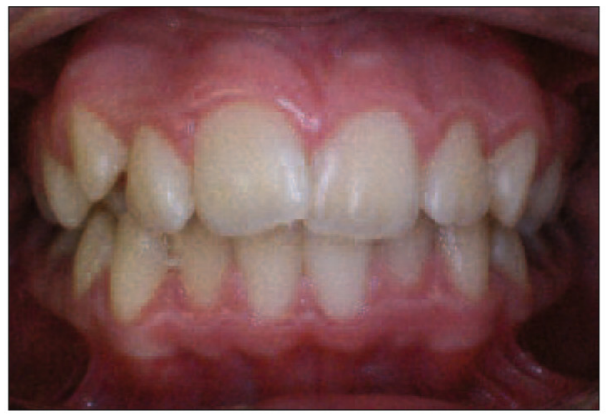

Figures 7 a to $c$

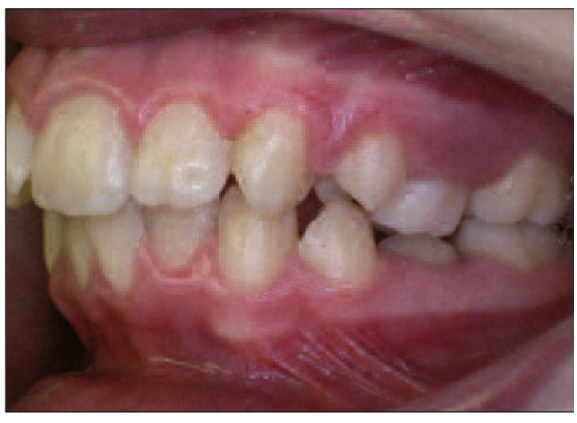

C

Intraoral views at the end of the first stage of treatment.

- both upper and lower arch width increased because of the improved position and activity of the tongue.

\section{Phase II}

Patient wore a "Multi P high $5^{\circledR}$ "' eruption guide for 9 months.

In this stage, the increased thickness of the appliance adjusted dento-alveolar growth in the posterior regions by limiting eruption of buccal teeth (fig. 8 to 11).

\section{Results :}

- lip competence improved to an acceptable level as patient received instruction in proper nasal respiration ;

- the width of both upper and lower arches continued to increase ;

- vertical dimension had been restored to an acceptable level.

\section{Phase III}

In two years of full banded and bonded orthodontic treatment 


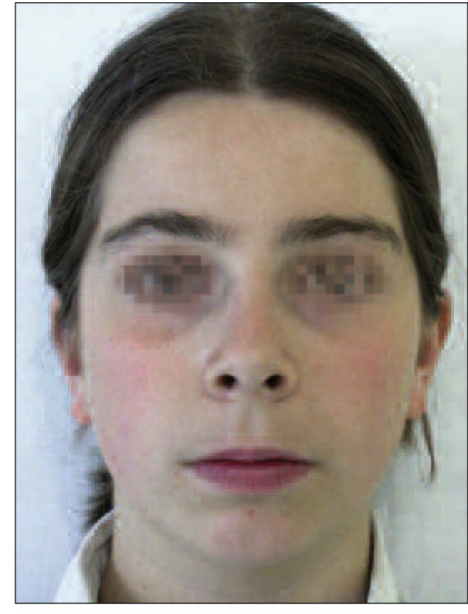

a

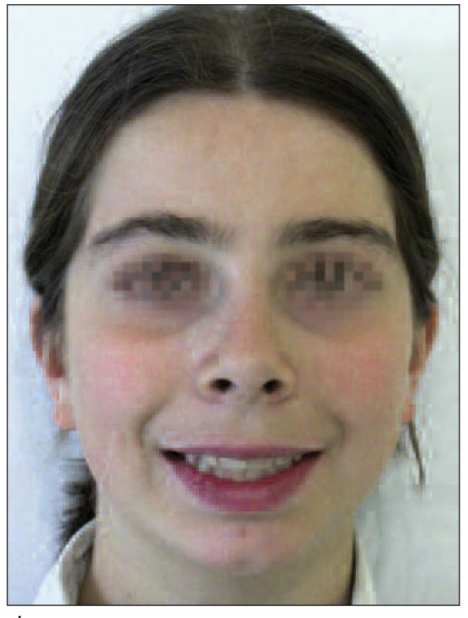

Figures 8 a to $c$

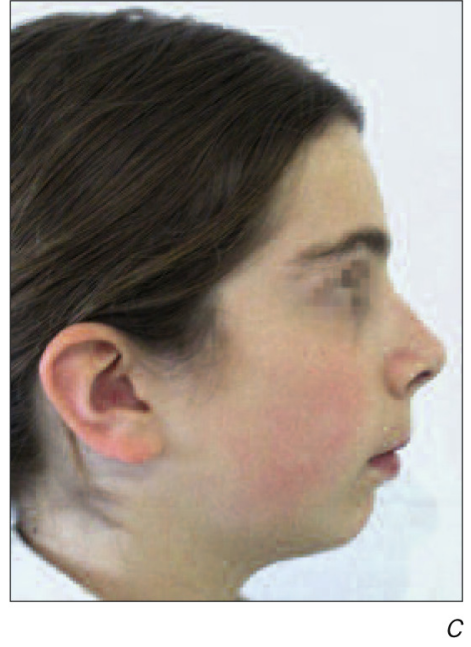

Facial photographs at the end of phase II.

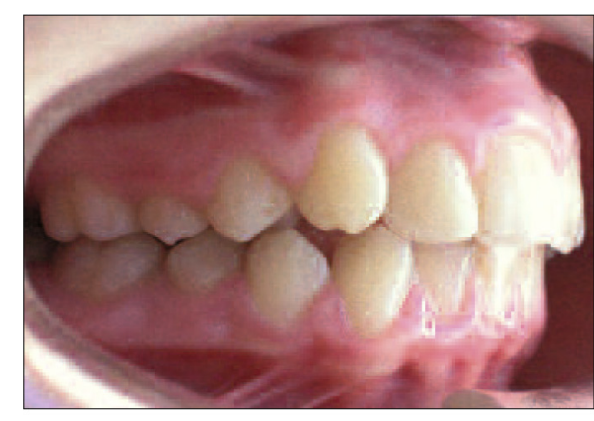

a

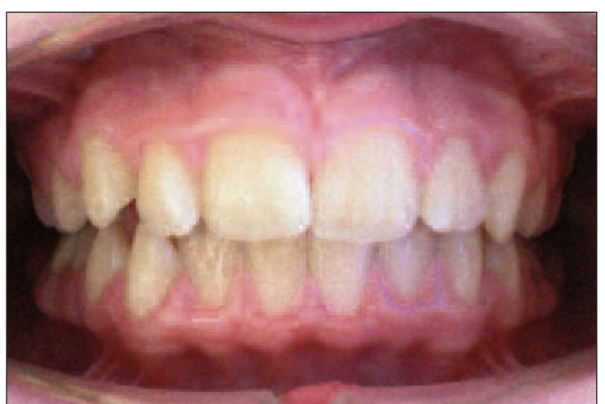

$b$

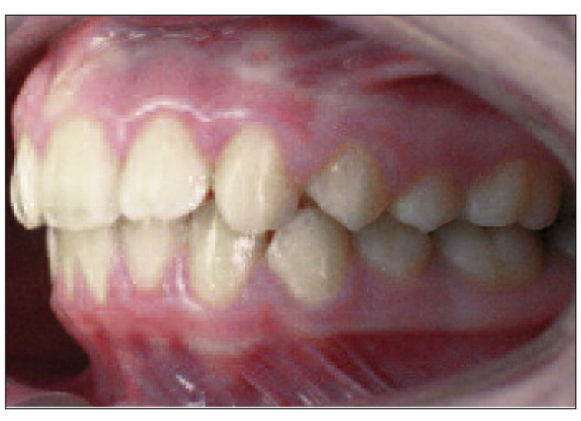

C

Figures 9 a to $c$

Intraoral views at the end of phase II.

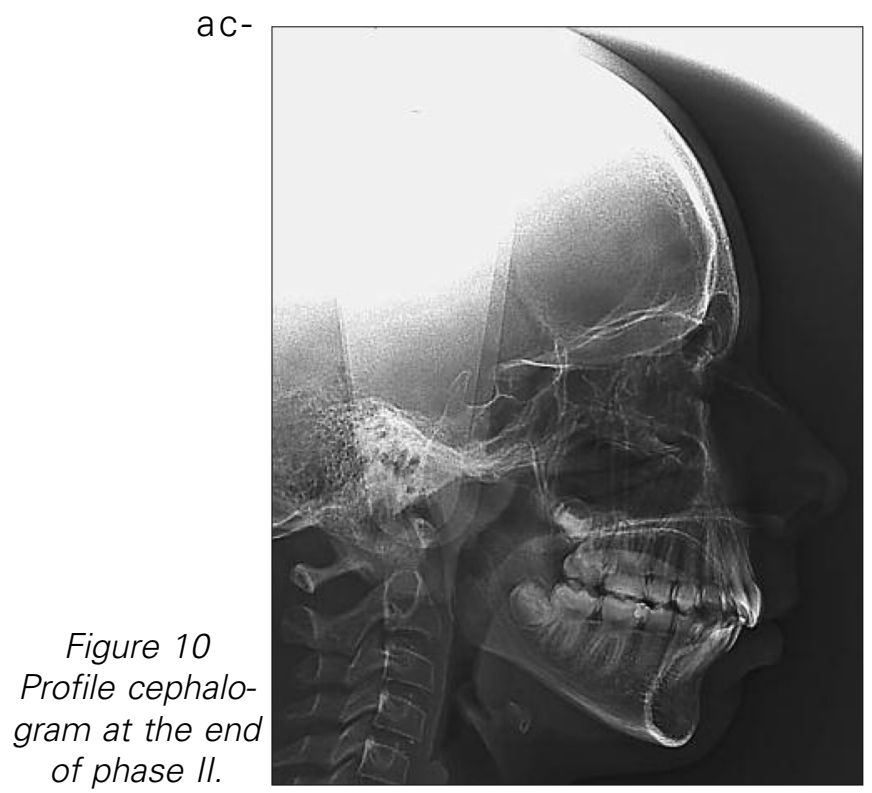

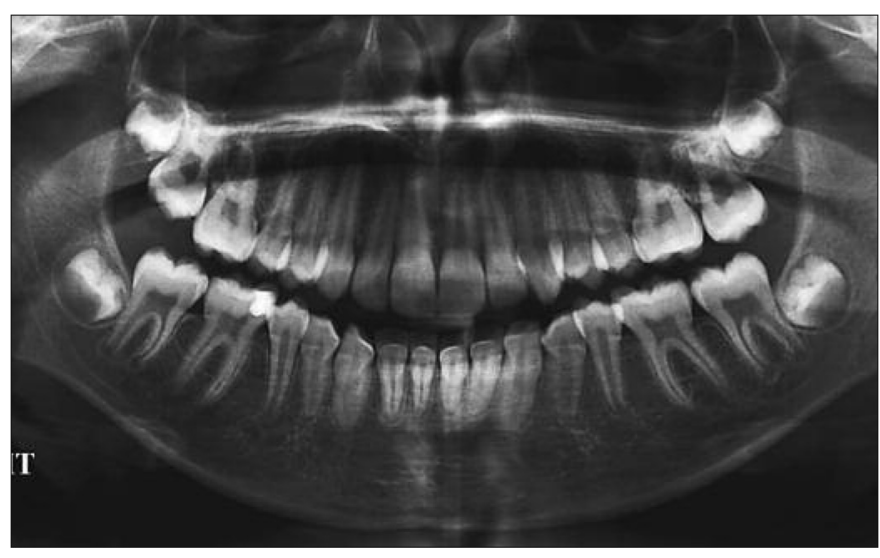

Figure 11

Panoramic X-ray at the end of phase II. 


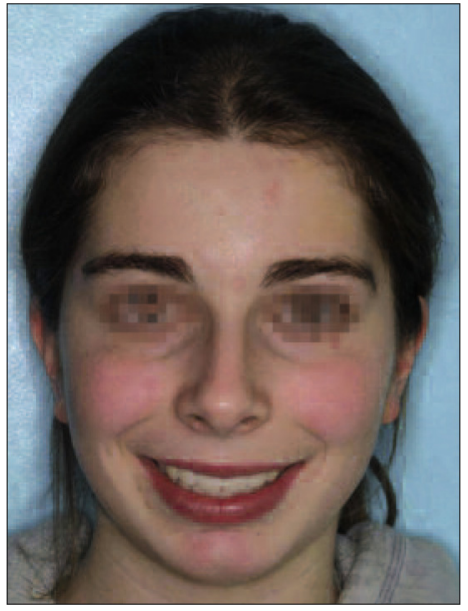

Figure 12

Smiling full face photo at the end of phase III.

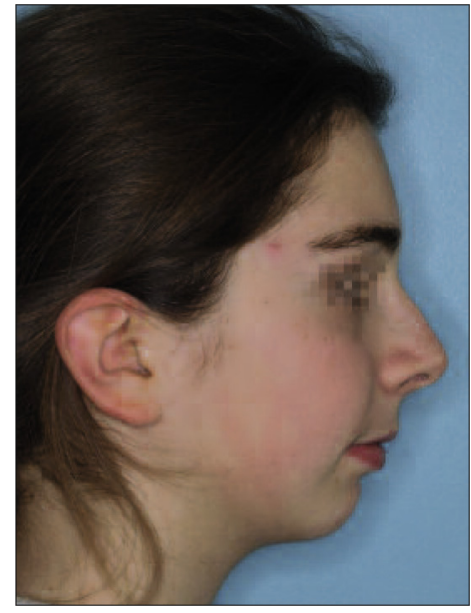

Figure 13

Profile photo at the end of phase III.

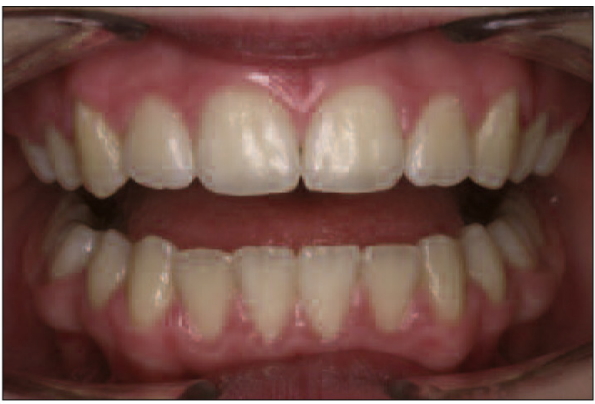

$a$

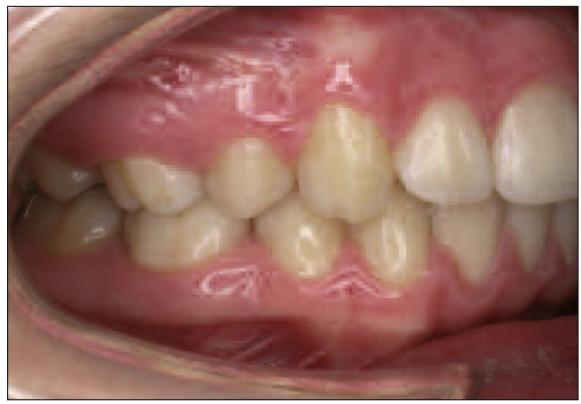

$b$

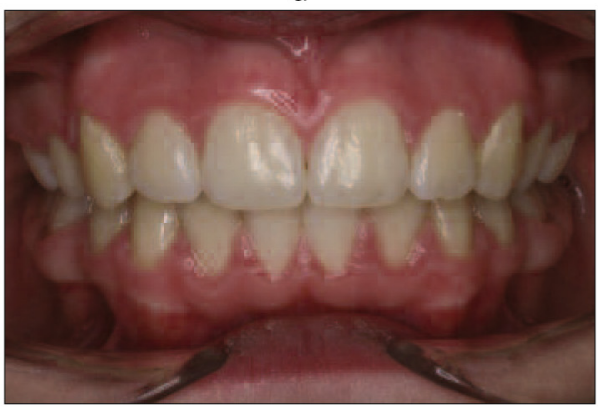

C

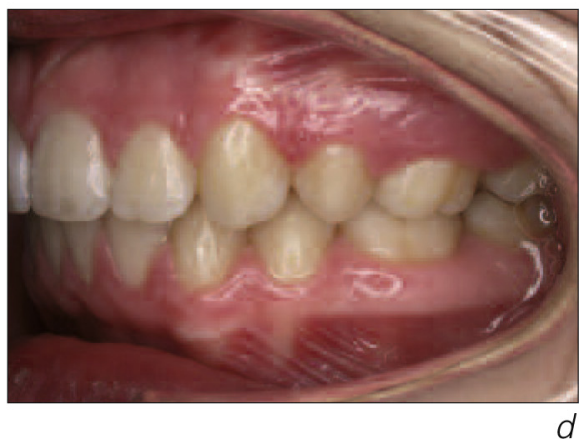

Figures 14 a to $d$

Intraoral views at the end of phase III. 
Figure 15 Profile cephalometric $X$-ray at the end of phase III.
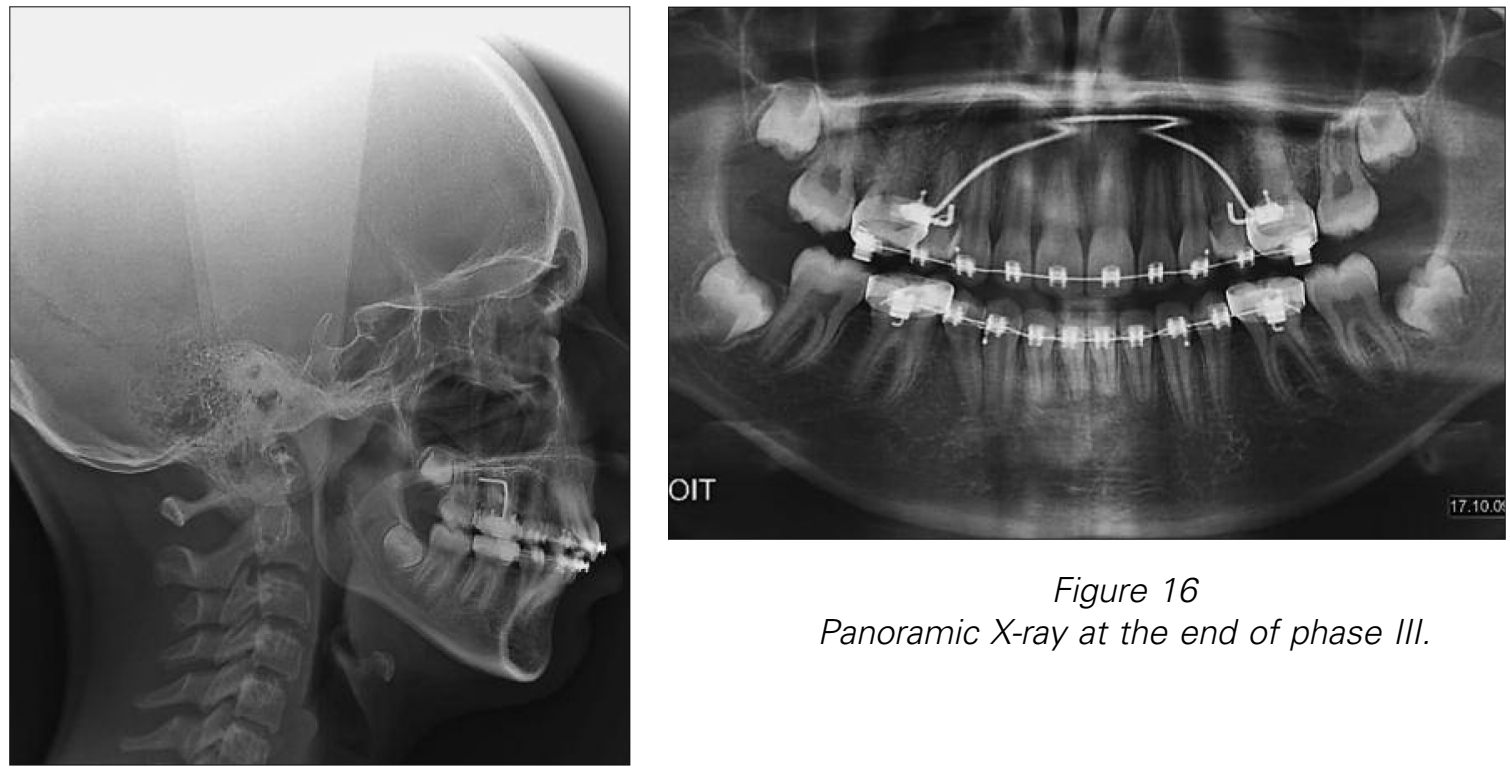

Figure 16

Panoramic X-ray at the end of phase III.

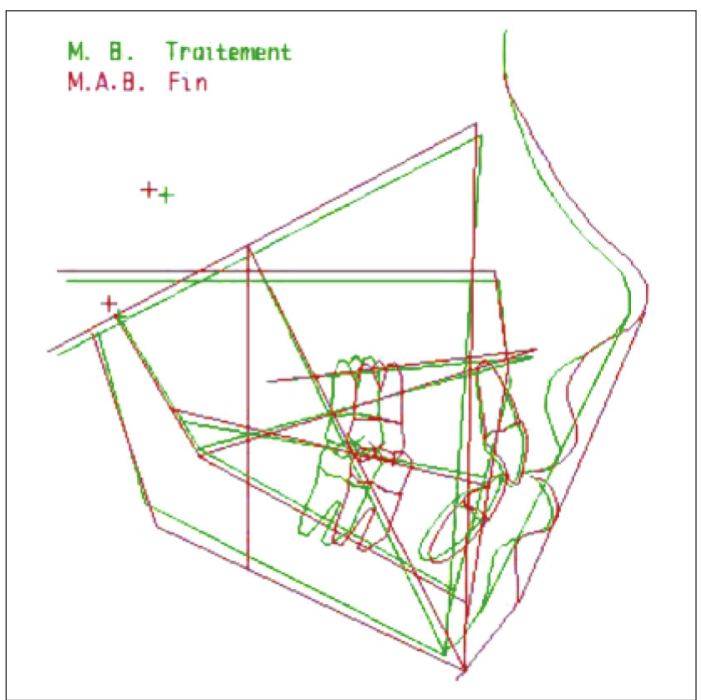

Figure 17

Superimposition of cephalometric tracings. 
companied by the extraction of the four second bicuspids and the use of a trans-palatal arch we reduced the bimaxillary protrusion and corrected the asymmetry while keeping vertical dimension within an acceptable range (fig. 12 to 17).

\section{CONCLUSION}

Midway through the 1960s orthodontic pioneers Daniel Subtelny, Robert Ricketts, and Donald Woodside began in-depth studies of the skeletal development of the face. Subtelny in particular demonstrated the essential role that poor functioning plays in the creation of skeletal malformations.

Treatment of hyperdivergent patients still presents a distinct challenge to orthodontists because of the number and importance of functional factors associated with it, including allergies and tempero-mandibular joint and respiratory problems. These discrepancies force functional adaptions like low tongue posture and mouth breathing that provoke chaotic interference with normal growth of skeletal structures in all three dimensions of space.

In these cases myo-functional therapy can play the key role of convincing

\section{Results :}

- myo-functional therapy restored proper functioning

- mechano-therapy achieved good occlusion and decrease of the bi-maxillary protrusion.

the doctor-patient-parent team how important it is to constitute a biologically informed plan as early as possible to prevent the inception and the development of malfunctioning that can have a three-dimensional unhealthy impact on skeletal structures. The successful unfolding of such a project will make it possible for orthodontists to later undertake a full banded and bonded treatment under improved functional conditions.

It is important to note that if the myo-functional therapy does not fully achieve its anticipated objectives this failure will be a warning signal for practitioners to re-evaluate the patient's records and to re-constitute their orthodontic orthodontic-surgical treatment plans with the aim of establishing objectives that are more objective and, if necessary, a compromise with the ideal. 